\title{
Stringent limitations on reductive perturbation studies of nonplanar acoustic solitons in plasmas
}

\author{
Frank Verheest ${ }^{1,2}$ and Manfred A. Hellberg ${ }^{2}$ \\ ${ }^{1}$ Sterrenkundig Observatorium, Universiteit Gent, Krijgslaan 281, B-9000 Gent, Belgium \\ ${ }^{2}$ School of Chemistry and Physics, University of KwaZulu-Natal, Durban 4000, South Africa
}

(Received 3 April 2016; accepted 7 June 2016; published online 22 June 2016)

\begin{abstract}
More than fifty years ago, the Korteweg-de Vries equation was shown to describe not only solitary surface waves on shallow water, but also nonlinear ion-acoustic waves. Because of the algorithmic ease of using reductive perturbation theory, intensive research followed on a wide range of wave types. Soon, the formalism was extended to nonplanar modes by introducing a stretching designed to accommodate spherically and cylindrically symmetric ion-acoustic waves. Over the last two decades many authors followed this approach, but almost all have ignored the severe restrictions in parameter space imposed by the Ansatz. In addition, for other steps in the formalism, the justification is often not spelled out, leading to effects that are physically undesirable or ambiguous. Hence, there is a need to critically assess this approach to nonplanar modes and to use it with the utmost care, respecting the restrictions on its validity. Only inward propagation may be meaningfully studied and respect for weak nonlinearities of at most $1 / 10$ implies that one cannot get closer to the axis or centre of symmetry than about 30 Debye lengths. Thus, one is in a regime where the modes are quasi-planar and not particularly interesting. Most papers disregard these constraints and hence reach questionable conclusions. Published by AIP Publishing.

[http://dx.doi.org/10.1063/1.4954308]
\end{abstract}

\section{INTRODUCTION}

After it was shown more than half a century ago that the Korteweg-de Vries (KdV) equation ${ }^{1}$ not only describes solitary surface waves on shallow water, but also nonlinear ionacoustic waves, ${ }^{2}$ the research in this domain has been intense. A great variety of plasma models and compositions has thus been covered, as well as various extensions to other members of the $\mathrm{KdV}$ family of nonlinear evolution equations. The relative success of reductive perturbation theory in describing nonlinear wave problems lies in the algorithmic ease of use, based on a separation of fast and slow timescales and of linear and nonlinear effects. This ideally leads to a balance between nonlinearity and dispersion, enabling the emergence of stable solitary structures that propagate unchanged. In addition to these solitary waves propagating without change in their speed and shape, KdV solitons show remarkable interaction properties, surviving overtaking of slower by faster solitons almost unchanged, and having characteristic relations between amplitude, width, and propagation speed. ${ }^{3}$

This explains the great attraction and the rapid and ongoing expansion in the number of papers devoted to this type of research. However, there are limitations and restrictions that have sometimes been overlooked. It is obvious that reductive perturbation theory rests on two pillars: a proper choice of the stretching used to rearrange the independent variables and a suitable expansion of the dependent variables. However, rather than simply positing a stretching + expansion scheme as many authors routinely do, and checking to lowest (linear) order in the expansion that all is well, we need to remember and stress that it is the linear dispersion properties which govern the choice of stretching. ${ }^{4}$ In principle, dispersion relations should therefore be determined first, before any stretching is chosen. Furthermore, the stretching determines the form of the evolution equation which one obtains.

Because of the success of KdV theory in studying nonlinear plane waves, it was natural that an attempt to extend the formalism to nonplanar wave studies would follow. In an initial pair of papers, Maxon and Viecelli ${ }^{5,6}$ introduced a form of stretching which they applied to the study of spherically and cylindrically symmetric ion-acoustic waves, respectively. Particularly over the last decade or so, these were followed by a flood of papers using their approach, whether actually citing their papers or not. In these, the authors applied the technique to nonlinear waves in a variety of multi-species plasmas having different properties.

It is important to note that there have been several experimental observations, initially by Hershkowitz ${ }^{7}$ and subsequently, particularly, by Nakamura and co-workers, of what are interpreted as cylindrical or spherical solitons. ${ }^{8-11}$ Here, we will use "soliton" as a shorthand for nonlinear, supersonic waves which preserve their shape, possibly with variations in amplitude, as they propagate. However, it must be borne in mind that the experimental evidence for this important property is difficult to assess and not fully convincing. The same goes for the interpretation of other details of the experiments in terms of the theoretical predictions of the Maxon-Viecelli theory. ${ }^{5,6}$

Unfortunately, close scrutiny of the Maxon-Viecelli approach indicates that it includes some steps which restrict its validity to a very limited range of parameter values. This aspect has inevitably been ignored in later papers, whether 
the authors cite the original papers or not. There are other steps in the formalism, justification for which is often not spelled out, which lead to effects that appear to be unphysical.

In trying to understand the methodology of Maxon and Viecelli, and hence also that of their followers, we were struck by many assumptions and ambiguities, and illustrated this on a selection of such references, ${ }^{12}$ including the original papers by Maxon and Viecelli. ${ }^{5,6}$ None of the authors other than Maxon and Viecelli even tried to justify their approach, a majority do not even acknowledge the sources for this stretching, obviously considering it as being on par with the plane-wave stretching of Washimi and Taniuti, ${ }^{2}$ often also without explicit reference to the originators. Almost all forget the severe restrictions mentioned by Maxon and Viecelli on its validity. ${ }^{6,13,14}$ Even after our focus on ambiguities and assumptions, ${ }^{12}$ the stream of submissions without proper justification and recognition of the resultant limitations imposed by the stretching seems to continue unabated.

Hence, there is a need in this mini-tutorial to show and prove in an unequivocal way that the Maxon-Viecelli stretching is to be used with the utmost care and respect for its domain of validity.

\section{BASICS OF REDUCTIVE PERTURBATION THEORY}

Our discussion will be based on the simplest archetypal acoustic wave, the ion-acoustic wave in a plasma composed of Boltzmann electrons and cold fluid singly charged ions, which is the model used in the original papers. ${ }^{5,6}$ At the risk of repeating well known knowledge, we note that the ionacoustic dispersion relation is ${ }^{15}$

$$
\frac{\omega^{2}}{k^{2}}=\frac{c_{i a}^{2}}{1+k^{2} \lambda_{D}^{2}} .
$$

Here, $\omega$ and $k$ refer to the (angular) frequency and wavenumber of the linear harmonic wave, respectively, $c_{i a}$ is the characteristic ion-acoustic speed, and $\lambda_{D}$ the (electron) Debye length. ${ }^{15}$ For very long wavelengths, for which $k \lambda_{D} \rightarrow 0$, but $\omega / k$ is kept finite, the acoustic modes are found with constant phase speed $\omega / k=c_{i a}$, and they are thus dispersionless. For slightly shorter wavelengths, but still satisfying $k \lambda_{D} \ll 1$, (1) can be approximated by

$$
\omega=k c_{i a}\left(1-\frac{1}{2} k^{2} \lambda_{D}^{2}\right),
$$

where we have chosen the sign for a propagation velocity $+c_{i a}$ in the positive $x$-direction. ${ }^{4}$ This leads to the typical phase argument for one-dimensional propagation

$$
k x-\omega t=k\left(x-c_{i a} t\right)+\frac{1}{2} k^{3} c_{i a} \lambda_{D}^{2} t,
$$

which in turns yields the ubiquitous KdV stretching ${ }^{2,4,16}$

$$
\xi=\varepsilon^{1 / 2}\left(x-c_{i a} t\right), \quad \tau=\varepsilon^{3 / 2} t .
$$

Taking, for example, the expansion of the electrostatic potential as $\phi=\varepsilon \phi_{1}+\varepsilon^{2} \phi_{2}+\cdots$, and going through the motions, the relevant $\mathrm{KdV}$ equation is readily obtained,

$$
A \frac{\partial \phi_{1}}{\partial \tau}+B \phi_{1} \frac{\partial \phi_{1}}{\partial \xi}+C \frac{\partial^{3} \phi_{1}}{\partial \xi^{3}}=0 .
$$

The first term gives the slow-time variation, the middle term the nonlinear effect, and the last one the dispersion.

The coefficients $A, B$, and $C$ are functions of the wave characteristics and plasma compositional parameters, and can be quite involved for more complicated plasma models, but the difficulties are algebraic rather than analytical in nature. From (5), it follows that, ignoring the slow timevariation, nonlinearity is balanced by dispersion in $\mathrm{KdV}$ structures.

Linearizing (5) for plane harmonic waves with phase $K \xi-\Omega \tau$ yields $\Omega=(C / A) K^{3}$, showing again the connection to the linear dispersion relation. In other words, while the stretching reflects the linear dispersion properties and the slow time variation, the structure of the nonlinearity is a child of the chosen expansion. As the literature abundantly testifies, many variations on this theme can be investigated, referring to other plasma compositions and/or classes of (nonlinear) waves.

This is all well and good, but it only works in a straightforward way for one-dimensional propagation, in which all variables are functions of only one space coordinate (and time), so that one obtains in essence the nonlinear equivalents of plane wave structures propagating in one direction, rather than expanding or contracting spherical or cylindrical waves. Clearly, it would be highly desirable to get away from the ubiquitous plane wave structures and investigate nonplanar waves.

\section{LINEAR WAVES}

As we have pointed out above, the linear wave underpins the choice of stretching that is often used to study the nonlinear problem. For clarity, we shall briefly list the basic equations for one-dimensional electrostatic waves in a plasma with cold fluid protons and Boltzmann electrons, ${ }^{5}$ in the usual ion acoustic normalized form ${ }^{12}$

$$
\begin{gathered}
\frac{\partial n}{\partial t}+\frac{1}{r^{\nu}} \frac{\partial}{\partial r}\left(r^{\nu} n u\right)=0, \\
\frac{\partial u}{\partial t}+u \frac{\partial u}{\partial r}+\frac{\partial \varphi}{\partial r}=0, \\
\frac{1}{r^{\nu}} \frac{\partial}{\partial r}\left(r^{\nu} \frac{\partial \varphi}{\partial r}\right)+n-\exp (\varphi)=0,
\end{gathered}
$$

with $\nu=0,1,2$ representing plane, cylindrically symmetric and spherically symmetric waves, respectively. Here, $n$ and $u$ refer to the ion density and fluid velocity, normalized to the undisturbed ion (and electron) density $n_{0}$ and the ion-acoustic velocity $c_{i a}=\sqrt{T_{e} / m_{i}}$, respectively, in terms of the electron kinetic temperature $T_{e}$ and ion mass $m_{i}$. Further, $r$ and $\varphi$ are the spatial coordinate and electrostatic potential, respectively, normalized to the electron Debye length $\lambda_{D}=\sqrt{\varepsilon_{0} T_{e} / n_{0} e^{2}}$ 
and to $T_{e} / e$. This leaves time $t$ normalized to the inverse of the ion plasma frequency $\omega_{p i}=\sqrt{n_{0} e^{2} / \varepsilon_{0} m_{i}}$.

We note from (6)-(8) that, in principle, the nonplanar geometry $(\nu=1,2)$ plays an explicit role in both the continuity equation and Poisson's equation, but not in the equation of motion.

\section{A. Some basic wave concepts}

Let us return to some of the basic assumptions about the plasma and possible plane or nonplanar modes. First of all, the plasma is homogeneous and stationary in the undisturbed conditions and the equations describing its motion are autonomous in time $t$, meaning that $t$ only occurs implicitly, through the dependent variables and their derivatives. This immediately allows us to choose the origin of timekeeping wherever we wish, as we see fit, and it also follows that after linearization of the dependent variables around their undisturbed values, the basic equations can be Fourier analyzed with respect to time in a useful way.

On the other hand, the basic equations are only autonomous in the spatial coordinate(s) as long as we work in Cartesian reference frames, which is specifically the case for plane waves propagating in a fixed direction. In a magnetized plasma, for instance, this may relate to a plane wave propagating at a fixed angle to a uniform magnetic field. However, whenever there are powers or functions of a space coordinate multiplying functions and derivatives of the dependent variables, a Fourier transform will lead to a convolution integral, ${ }^{17}$ and this is not useful if one wants to determine a dispersion relation.

This typically occurs when one investigates nonplanar plasma modes in cylindrical or spherical coordinates, even when angular effects are not considered because of cylindrical or spherical symmetry with respect to an axis or a centre of symmetry, respectively. In that case, $r$ is the radial distance from the axis or from the centre and, even after linearizing the dependent variables, there is no point in Fourier analyzing with respect to $r$. Hence, all notions of obtaining a linear dispersion relation of the standard form, $D(\omega, k)=0$, are lost, unless great care is taken to observe stringent limitations on the radial domain, as discussed below.

\section{B. Blindly using Fourier analysis}

Let us see what happens if, despite the general comments above, one decides to blindly carry out a Fourier analysis of (6)-(8). This approach has been discussed in our earlier paper, ${ }^{12}$ and we recall some of those results that illustrate graphically what follows.

In the usual way, we substitute $n=1+\varepsilon n_{A} \exp$ $[i(k r-\omega t)], \quad u=\varepsilon u_{A} \exp [i(k r-\omega t)], \quad$ and $\varphi=\varepsilon \varphi_{A} \exp$ $[i(k r-\omega t)]$, for $\varepsilon$ small. Obviously, the amplitudes $n_{A}, u_{A}$, and $\varphi_{A}$ must be independent of the independent variables of the original equations, $r$ and $t$, as well as of $k$ and $\omega$.

One then finds that the ion density satisfies

$$
n_{A}=\frac{k^{2}}{\omega^{2}}\left(1-\frac{i \nu}{k r}\right) \varphi_{A},
$$

leading to the linear dispersion relation

$$
\frac{\omega^{2}}{k^{2}}=\left(1-\frac{i \nu}{k r}\right) /\left[1+k^{2}\left(1-\frac{i \nu}{k r}\right)\right] .
$$

Hence, $V^{2}=\lim _{k \rightarrow 0}(\omega / k)^{2}$ should yield the linear acoustic velocity to be used as the basis of the stretching.

For strictly plane waves, $\nu=0$ simplifies the expression (10) so that taking the limit $k \rightarrow 0$ of $\omega / k$ yields $V=1$, and one finds the familiar plane-wave stretchings. ${ }^{2}$

However, for nonplanar modes $(\nu \neq 0)$, there is evidently a major difficulty in (10), namely, the appropriate "acoustic speed" is $r$-dependent. Hence, the harmonic linear wave method, having assumed constant $\omega$ and $k$, only works when the imaginary terms $i \nu / k r$ disappear. These terms are bothersome not so much because they are imaginary, but because the frequency is now found to be spatially varying for real $k$. It might appear that a possible way out would be to assume that the terms in $\nu / k r$ are small enough so as to be able to neglect them. However, computing $\lim _{k \rightarrow 0}(\omega / k)$ would then amount to requiring that $r \rightarrow \infty$ faster than $k \rightarrow 0$. Thus, for general radial positions $r$, one cannot find an acceptable value for $V$, despite claims to the contrary. $5,6,13,14$

A part of this argument was already contained in our earlier paper, ${ }^{12}$ which has been studiously ignored, except for a recent paper by Shan and Rehman ${ }^{18}$ who effectively repeated a part of our text, without taking account of the consequences that follow, and in fact without proper reference initially. Subsequently, this was partially rectified in an Erratum. ${ }^{19}$

We also note that for linear modes containing an extra factor $1 / r$ upfront, as for spherical electromagnetic waves, the $r$-dependent imaginary terms in (10) are eliminated for $\nu=2$, but other equally serious $r$ dependences appear. Indeed, the result is then

$$
\frac{\omega^{2}}{k^{2}}=\frac{1+k^{2} r^{2}}{k^{2} r^{2}\left(1+k^{2}\right)} \text {. }
$$

Thus, however one approaches the problem, the assumed linear wave behaviour, the basis of any possible stretching, is valid only for $k r \gg 1$, together with $k \ll 1$.

\section{Aspects of the standard wave equation}

It can easily be shown that, without some further approximation, the basic fluid equations (6) and (7), coupled by Poisson's equation (8), do not lead to a wave equation of standard form in nonplanar geometry. Nonetheless, let us next remind ourselves of waves that can be described by a wave equation in a single dependent variable $v$

$$
\nabla^{2} v-\frac{1}{c^{2}} \frac{\partial^{2} v}{\partial t^{2}}=0
$$

with $c$ as the characteristic propagation velocity.

In Cartesian coordinates, the solution of (12) is well known to be

$$
v(\mathbf{r}, t)=F(\mathbf{k} \cdot \mathbf{r} \pm \omega t) .
$$

Here, $F$ is an arbitrary function of its phase argument $\mathbf{k} \cdot \mathbf{r} \pm \omega t, \mathbf{k}$ is the wave vector giving the direction of propagation, and $\omega=\|\mathbf{k}\| c$ is the (angular) frequency. Thus, the 
shape of the wave is arbitrary, and wave propagation occurs at constant speed, $c=\omega /\|\mathbf{k}\|$, without a change in amplitude or shape occurring.

For cylindrical or spherical waves, propagating in/outward from the axis or centre of symmetry, the wave equation becomes

$$
\frac{1}{r^{\nu}} \frac{\partial}{\partial r}\left(r^{\nu} \frac{\partial v}{\partial r}\right)-\frac{1}{c^{2}} \frac{\partial^{2} v}{\partial t^{2}}=0
$$

We can see immediately that, because of the factors $r^{\nu}$, the equation is no longer autonomous in $r$.

Fortunately, for spherical waves $(v=2)$, it can be transformed into an autonomous equation in $r v$, using the property that

$$
\frac{1}{r^{2}} \frac{\partial}{\partial r}\left(r^{2} \frac{\partial v}{\partial r}\right)=\frac{1}{r} \frac{\partial^{2}}{\partial r^{2}}(r v),
$$

and after subsequent multiplication by $r$ one obtains

$$
\frac{\partial^{2}}{\partial r^{2}}(r v)-\frac{1}{c^{2}} \frac{\partial^{2}}{\partial t^{2}}(r v)=0
$$

This yields the standard spherical wave solutions

$$
v=\frac{1}{r} F(r \pm c t),
$$

well-known from electromagnetic theory, ${ }^{20}$ where $v$ represents one of the components of the electric or magnetic fields. Thus, a wave of arbitrary shape will expand or contract radially at constant speed $c$. However, the amplitude diminishes as $1 / r$ due to conservation of total wave energy. It follows that, subject to the caveat regarding the diminishing amplitude, one can, for the spherically symmetric wave, find a wave frame moving with constant speed, in which linear waves of arbitrary shape will propagate without a change of shape.

Importantly, no analogous cylindrical wave solutions can be found, when $\nu=1$. In fact, as can be checked, shapepreserving symmetric solutions can be obtained in $n-$ dimensional curvilinear coordinates only for the spherical case, $\nu=2$, albeit with a radially decreasing amplitude as seen above. In any other number of dimensions, the shape of an arbitrary wave is not preserved. ${ }^{21}$ Thus, it follows that, in the case of cylindrically symmetric waves, it is not in general possible to move to a wave frame moving with fixed speed without some distortion occurring.

Specifically, it is well known that the radial part of the solutions to the cylindrically symmetric wave equation is given by Hankel functions of order zero, ${ }^{9,22,23}$ usually denoted by $H_{0}^{1}(k r)$ and $H_{0}^{2}(k r)$, respectively.

Fortunately, the cylindrically symmetric case can be saved in the asymptotic limit $k r \gg 1$. In that limit, $H_{0}^{1}(k r)$ and $H_{0}^{2}(k r)$ vary as $r^{-1 / 2} \exp ( \pm i k r)$, respectively. ${ }^{22,23}$ Thus, a harmonically varying solution $\left(\propto r^{-1 / 2} \exp [i(\omega t \pm k r)]\right)$ can be obtained. Although one is restricted to harmonic waves (which are, of course, of fundamental importance), at least such waves will propagate at constant speed with constant shape, albeit with a radially varying amplitude. Analogously to the spherical case, a fore-factor $\left(r^{-1 / 2}\right.$ in this case) is required to ensure that total wave energy is conserved as the wave expands or contracts radially.

In summary then, solution of the cylindrically symmetric wave equation allows one, only in the limit $k r \gg 1$, to move to a wave frame within which harmonic waves propagate with unchanged shape (constant wavelength), but with radially varying amplitude. We must emphasize, however, that this underlying assumption is not normally discussed in the many papers in the standard literature on cylindrically symmetric soliton propagation. We note that Refs. 6, 13, and 14 , although not commenting on $k r$ explicitly, do mention that the approach is valid only for large $r / \lambda_{D}$.

\section{Quasineutrality}

Before proceeding to investigate a further fundamental assumption, we emphasize again that, unfortunately, the basic fluid and Poisson's equations in electrostatic plasma problems cannot, in general, be reduced to a single wave equation of the form given above in (14), but they remain intrinsically non-autonomous, even after linearization of the dependent variables.

An exception to this statement arises when one introduces the assumption of quasineutrality, that is, one ignores Poisson's equation and instead sets the ion density equal to the electron density. It is well-known ${ }^{15}$ that this additional physical assumption of ignoring the effects of charge density fluctuations implies that one is restricted to considering waves with wavelengths longer than the Debye length, specifically satisfying $k^{2} \ll 1$ (normalized to $\lambda_{D}$ ), as one may see from (1). In that case, the basic equations (6)-(8) are reduced to

$$
\begin{gathered}
\frac{\partial \delta n}{\partial t}+\frac{1}{r^{\nu}} \frac{\partial}{\partial r}\left(r^{\nu} \delta u\right)=0, \\
\frac{\partial \delta u}{\partial t}+\frac{\partial \delta \varphi}{\partial r}=0, \\
\delta n-\delta \varphi=0 .
\end{gathered}
$$

Taking the time derivative of (18) and eliminating $\partial \delta u / \partial t$ with the help of (19) leads to the standard wave equation, (14), for arbitrary $\nu$, with the dependent variable satisfying either $v=\delta \varphi$ or $\delta n$ and $c=1$. Nakamura and Ogino ${ }^{10,11}$ state that $\delta n$ satisfies an equation of this form for nonplanar ion-acoustic solitons. However, they unfortunately do so without providing any details on how their result is achieved and specifically not drawing attention to the limitation imposed.

Following (17) for spherical waves $(\nu=2)$, one can show that

$$
\delta n=\delta \varphi=\frac{1}{r} F(r \pm t) .
$$

Unfortunately, however, this is only partially helpful as one can readily show that the expression for $\delta u$ is not of the same form. The full problem of interest is, of course, actually 
nonlinear, and in the standard $\mathrm{KdV}$ expansion, one normally finds that, to linear order, the independent variables satisfy $\delta \varphi=\delta n=|\delta u|$. Hence, this result, although useful at first sight, turns out to be disappointingly restricted.

\section{RE-DERIVING THE EXTENDED KORTEWEG-DE VRIES EQUATION}

\section{A. Evolution equation}

In Sec. III B, strong indications emerged that one will have to work at large $r$ in order to salvage a form of reductive perturbation theory when investigating nonplanar waves. Let us go back to the usual $\mathrm{KdV}$ equation (5), obtained using the stretching (4) (now with normalized $c_{i a}=1$ ). Its sechsquared soliton solution is traditionally pictured as moving slightly superacoustically from left to right. Because the basic equations in plane geometry are autonomous in $x$ and $t$, the origin of space and time can be chosen at will, as are the orientations of the axes. Thus, a soliton which is in some $x_{0}$ at $t_{0}$ travels to $x_{1}>x_{0}$ at $t_{1}>t_{0}$ at speed $1+w>1$. Hence, for the co-moving coordinate, we obtain

$$
x_{1}-x_{0}=(1+w)\left(t_{1}-t_{0}\right) \quad \Rightarrow \quad \zeta=\xi-w \tau,
$$

where $\xi=x-t$, setting the bookkeeping parameter $\varepsilon=1$ for the final evaluation.

In the nonplanar geometry we are discussing, the nonplanar modes will look more and more like plane waves, the larger $r$ becomes, and ideally so for $r \rightarrow+\infty$. It thus makes no sense to start with initial conditions at large $r$ and let the nonlinear structure move outward from the axis or origin of symmetry, as it will merely lose any nonplanar characteristics that it may have.

Therefore, we argue that one can only meaningfully investigate waves propagating inward, towards the axis or origin of symmetry. Obviously, the basic equations are now nonautonomous in $r$, and by definition $r \geq 0$. In other words, for inward propagation a solitary structure which is at position $r_{0}$ at time $t_{0}$ moves to $r_{1}<r_{0}$ at $t_{1}>t_{0}$. Now we have for the co-moving coordinate that

$$
r_{1}-r_{0}+(1+w)\left(t_{1}-t_{0}\right)=0 \Rightarrow \zeta=\xi+w \tau,
$$

provided the phase argument is chosen as $\xi=r+t$.

To see what the Maxon-Viecelli approach really implies, we follow their stretching for the independent variables, ${ }^{5,6,13,14}$ in the form

$$
\xi=\varepsilon^{1 / 2}(r+t), \quad \tau=\varepsilon^{3 / 2} t,
$$

coupled to the usual expansion of the dependent variables

$$
\begin{aligned}
n & =1+\varepsilon n_{1}+\varepsilon^{2} n_{2}+\cdots, \\
u & =\varepsilon u_{1}+\varepsilon^{2} u_{2}+\cdots, \\
\varphi & =\varepsilon \varphi_{1}+\varepsilon^{2} \varphi_{2}+\cdots
\end{aligned}
$$

Inserting (24) and (25) into (6)-(8) gives to lowest nonzero order

$$
\begin{gathered}
\frac{\partial n_{1}}{\partial \xi}+\frac{\nu}{r} u_{1}+\frac{\partial u_{1}}{\partial \xi}=0, \\
\frac{\partial u_{1}}{\partial \xi}+\frac{\partial \varphi_{1}}{\partial \xi}=0, \\
n_{1}-\varphi_{1}=0 .
\end{gathered}
$$

A first remark here is that if $r$ were of order unity, the middle term in (26) would be of order $\varepsilon$, lower than the two other terms with derivatives which are of order $\varepsilon^{3 / 2}$. Hence, the middle term would dominate and cannot be balanced, leading to $u_{1}=0$ and nothing remains. This means that $1 / r$ has to be at least of order $\varepsilon^{1 / 2}$, quantifying for the first time that one has to work at fairly large distances from the symmetry axis or origin.

Further, in (27), the two terms are of order $\varepsilon^{3 / 2}$, whereas in (28) the two lowest-order terms are of order $\varepsilon$, and corrections in $1 / r$ would appear only at higher order, even if $1 / r$ were of order unity. As a result, (28) reduces to a quasineutrality condition, $n_{1}=\varphi_{1}$. It is of interest to note that this is consistent with the requirements arising from the need to derive a linear wave equation from the basic plasma equations, as we have discussed in Sec. III D.

This notwithstanding, retaining the middle term in (26), with $1 / r \sim \varepsilon^{1 / 2}$, would lead to an analytical blockage, since no workable analysis exists to then determine the first order variables. The only way out is to restrict the treatment to even larger distances, in the sense that $\nu u_{1} / r$ becomes of order $\varepsilon^{5 / 2}$, and can thus be relegated to the continuity equation at that order. The consequence is that $1 / r$ has to be of order $\varepsilon^{3 / 2}$, a restriction mentioned already by Maxon and Viecelli. ${ }^{6,13,14}$ However, they do not base this restriction on the above argument. Instead, they point out that this ordering is required so that the ingoing cylindrical solitons, which travel towards increasing $\xi$, do not cross the singularity at $r=0 .{ }^{6,13,14}$ It is not clear how these two arguments are linked to one another. Importantly, this restriction on $r$ is often ignored in the numerical applications in the literature, particularly when authors allow for propagation too close to the axis or origin, including values of $r$ that are too small, as will be seen below.

Unexpectedly, in the Maxon-Viecelli formalism, the term in $1 / r$ is transformed into a $1 / \tau$ term. Thus, a spacelike term surprisingly becomes a time-like term without any explanation. A possible reason is found if one combines the stretching (24) with the ordering of $r$ so that one formally obtains $^{12,24}$

$$
r=\varepsilon^{-3 / 2}(\varepsilon \xi-\tau),
$$

which yields that $1 / r \sim-\varepsilon^{3 / 2}(1 / \tau)$, and requires that $\tau$ be negative. We note in passing that this can also be written as $r \sim-\varepsilon^{-3 / 2} \tau=-t$, a point that we shall return to in Sec. IV B.

However, substituting for $r$ in terms of $\tau$ as indicated above has several unintended and unfortunate consequences. First of all, it transforms what is a spatial limitation into a time limitation and thereby obscures the restrictions on $r$. Second, the original equations (6)-(8) are autonomous in $t$, 
meaning not only that their linearized form can be Fourier analyzed in time, but moreover, the homogeneity of time allows us to choose the origin of our timekeeping freely at will. Yet now, the $1 / \tau$ terms in the Maxon-Viecelli approach (faithfully followed by all the authors using their method) will blow up at the origin of timekeeping that we were supposed to be able to choose freely, without altering the physics. This is physically not consistent, so that we shall continue to write the terms in $\nu / r$ in that form, at least for the time being.

Returning now to (26), and neglecting the middle term in $\nu u_{1} / r$ as being of order $\varepsilon^{5 / 2}$, we arrive at the linear result that

$$
n_{1}=\varphi_{1}=-u_{1}
$$

The determination of $u_{1}$ involves an integration with respect to $\xi$, which we will assume to occur for zero undisturbed conditions infinitely far from the symmetry axis or origin. This result is, of course, the same as one finds for planar geometry, that is, it does not reflect any nonplanar characteristics at the linear level if $1 / r \sim \varepsilon^{3 / 2}$.

Turning our attention to the next significant order yields

$$
\begin{aligned}
& \frac{\partial n_{2}}{\partial \xi}+\frac{\partial n_{1}}{\partial \tau}+\frac{\nu}{r} u_{1}+\frac{\partial u_{2}}{\partial \xi}+\frac{\partial}{\partial \xi}\left(n_{1} u_{1}\right)=0, \\
& \frac{\partial u_{2}}{\partial \xi}+\frac{\partial u_{1}}{\partial \tau}+u_{1} \frac{\partial u_{1}}{\partial \xi}+\frac{\partial \varphi_{2}}{\partial \xi}=0 \\
& \frac{\partial^{2} \varphi_{1}}{\partial \xi^{2}}+n_{2}-\varphi_{2}-\frac{1}{2} \varphi_{1}^{2}=0
\end{aligned}
$$

In the last equation of (31), there should also be, in principle, a term $(\nu / r) \partial \varphi_{1} / \partial \xi$. However, if the reasoning is followed as when dealing with the linear equations, it would be of order $\varepsilon^{3}$, and thus has been omitted as being of higher order. It thus follows that even to this order, the expanded Poisson's equation does not exhibit any nonplanar characteristics, which are manifested only in the continuity equation.

Substituting (30) in this and eliminating all terms involving $n_{2}, u_{2}$, and $\varphi_{2}$ leads to an extended $\mathrm{KdV}(\mathrm{eKdV})$ equation

$$
\frac{\partial \varphi_{1}}{\partial \tau}-\varphi_{1} \frac{\partial \varphi_{1}}{\partial \xi}-\frac{1}{2} \frac{\partial^{3} \varphi_{1}}{\partial \xi^{3}}-\frac{\nu}{2 r} \varphi_{1}=0 .
$$

Bearing in mind the fact that we have made a number of approximations to reach this equation, it is important that we consider carefully its region of validity. This can be found by interpreting the $1 / r$ term as follows. For linear amplitudes of, say, $n_{1}=\varphi_{1} \simeq 1 / 10$, it follows from (29) that $r$ has to be larger than $r_{0} \simeq 30$ for this formalism to apply. Thus, outgoing solitons must start from $r_{0}$ and propagate to larger distances, whereas inward propagating solitons can only be validly described by (32), provided they get no closer than $r_{0}$ to the axis or origin of symmetry. We shall return to this important aspect below.

\section{B. Results and discussion}

It is well-known that, for $\nu=0$ (or formally also for $r \rightarrow+\infty$, although that limitation is not needed in pure plane geometry), one might adopt the standard one-soliton solution $^{4}$

$$
\varphi_{1}=3 w \operatorname{sech}^{2}\left[\sqrt{\frac{w}{2}}(\xi+w \tau)\right]
$$

where $w$ is the small excess over the linear acoustic speed, 1 in the chosen normalization. In the nonplanar geometry we are discussing, (33) is only a solution to (32) in the formal limit $r \rightarrow+\infty$. This is an indication that there is no point in using this expression as a starting point for the numerical simulations of outgoing waves, to see how these would evolve under (32) for even larger $r$. It thus underlines the fact that one should consider ingoing waves only.

There are further remarks on the motion of the centre of the solitary structure. Normal plots of (plane) solitons are drawn in a co-moving frame, where the structure is stationary, in other words, centered on $\zeta=0$. Bearing in mind the relation between the linear perturbations given by (30), together with (33), it follows that for typical linear amplitudes $\simeq 1 / 10$, the excess speed $w \simeq 1 / 30$. Hence, to a good approximation $\zeta=0$ is close to $\xi=0$, or $x \simeq|t|$ in plane geometry. Applying this reasoning also to nonplanar structures, we find that $r+t \simeq 0$, so that very large $r$ (intrinsically positive) corresponds to very negative $t$. This deduction is consistent with our observation in Sec. IV A that $r \simeq-t$.

Inward propagation can only be described by (32) as long as $r$ remains sufficiently large, and correspondingly, $t$ has to remain sufficiently negative. So, if we want to start from the planar solution and let that evolve under (32), we have thus determined a (qualitative) restriction on how large $|t|$ has to remain. This is the way out of the conundrum that, even though the basic equations are autonomous in $t$ and therefore one might a priori think of choosing the origin of $t$ at liberty, the conditions on suitable values of $r$, and hence on the proper use of (32), impose an origin for $t$ that may not be reached from large negative $t$. This constraint does not appear to have been discussed in the literature at all.

Once all this is clarified, a final sticking point remains. As derived, (32) is written in three variables, $\xi, \tau$, and $r$. For numerical computations, this is unworkable, and we propose to now eliminate $r$ by the replacement $r \rightarrow-\tau$, as all terms in (32) have been assumed to be of order $\varepsilon^{5 / 2}$. This brings one in line with what is usually found in the papers dealing with nonplanar solitons for the extended $\mathrm{KdV}$ equation

$$
\frac{\partial \varphi_{1}}{\partial \tau}-\varphi_{1} \frac{\partial \varphi_{1}}{\partial \xi}-\frac{1}{2} \frac{\partial^{3} \varphi_{1}}{\partial \xi^{3}}+\frac{\nu}{2 \tau} \varphi_{1}=0 .
$$

The important difference from the general literature is that we are now fully aware of how the ranges in $|\tau|$ and $r$ are limited by the strong constraint on the (relatively large) minimum distance from the axis or origin of symmetry that is permitted by the framework underpinning this equation.

In addition, ${ }^{25}$ we note that in (32), with $r$ appearing explicitly, the waves propagate in a region with variable curvature at each time, whereas in (34) the curvature does not appear explicitly and it is constant at each time, but changes with time. The only way in which this is acceptable is if the 
spatial extent of all the perturbations, $\delta r \ll r$. That is, (34) can only describe, e.g., in cylindrical coordinates, a narrow annular region where $\delta r / r \ll 1$ and cannot address the propagation of widely separated perturbations in the same domain. As perturbations move closer to $r=0$, this restriction becomes more severe. This is yet another rephrasing of arguments that were outlined earlier.

We would like to stress that the eKdV equation for the nonplanar case, given in (34), is not integrable in the usual understanding of this property. As discussed already in our previous paper, ${ }^{12}$ Infeld and Rowlands ${ }^{24}$ suggest that one multiply (34) by $\tau$, to obtain a continuity-like equation of the form

$$
\frac{\partial}{\partial \tau}\left(\varphi_{1} \tau\right)-\frac{\partial}{\partial \xi}\left[\frac{\tau}{2}\left(\varphi_{1}^{2}+\frac{\partial^{2} \varphi_{1}}{\partial \xi^{2}}\right)\right]=0 .
$$

This has been written for spherical modes, with $\nu=2$, to avoid discussions about the sign of $\tau$. Integration of (35) over all $\xi$, from $-\infty$ to $+\infty$, assuming that $\varphi_{1}$ and its derivatives vanish at $\xi= \pm \infty$, as we and all authors in this domain have done, leads to

$$
\int_{-\infty}^{+\infty} \frac{\partial}{\partial \tau}\left(\varphi_{1} \tau\right) d \xi=\frac{\partial}{\partial \tau}\left\{\tau \int_{-\infty}^{+\infty} \varphi_{1} d \xi\right\}=0 .
$$

Indeed, as $\xi$ and $\tau$ are independent variables, one can interchange the differentiation with respect to $\tau$ and the integration over all $\xi$, and also take $\tau$ out of this integration. As the integral $\int_{-\infty}^{+\infty} \varphi_{1} d \xi$ is carried out over all $\xi$, it follows that there is no $\xi$ dependence left, only a $\tau$ dependence implicitly through $\varphi_{1}$. However, the expression between curly brackets in (36) cannot depend on $\tau$, since its derivative with respect to $\tau$ vanishes. It must therefore be a pure constant, say, $C$, yielding that

$$
\int_{-\infty}^{+\infty} \varphi_{1} d \xi=\frac{C}{\tau}
$$

showing that the area under the curve $\varphi_{1}$ (the soliton profile) vanishes as $|\tau| \rightarrow \infty$ and blows up as $\tau \rightarrow 0$.

This is clearly not a constant of the motion in the usual sense. Therefore, multi-soliton solutions, infinite series of conserved densities, the emergence of solitons from an initial perturbation, and the like, do not exist, quite contrary to what happens for the standard (planar) KdV equation. This renders the choice of an initial profile in the numerical analysis of the solutions of (34) of the utmost importance, as discussed below.

In our earlier paper, ${ }^{12}$ we discussed a selection of papers [Refs. 12-14 and 16-56 therein] in order to examine the extent to which the underlying restrictions on the use of the Maxon-Viecelli method were heeded, e.g., keeping the amplitudes sufficiently small. Now that we have a clearer picture of the different limitations, we have done two things: first, revisited the original references in our earlier paper, ${ }^{12}$ and second, included a selection of the recent literature published in 2014 and 2015. ${ }^{18,26-42}$

Because we have dealt with a simple model for ionacoustic nonplanar modes, leading to a modified $\mathrm{KdV}$ equation (32) or (34), we have restricted the 2014-2015 selection to those papers that also derived nonplanar evolution equations of the KdV family, ${ }^{18,26,29,33,35-38,40,42}$ including the Gardner equation (KdV with quadratic and cubic nonlinearities), ${ }^{30,32,39,41}$ and, at the limit, the KdV-Burgers equation..$^{27,28,31,34}$ Needless to say, most of these papers have investigated plasma compositions more complicated than our simple model, but have derived equations similar to (34), with more intricate coefficients. Our focus on the simplest plasma model, that of cold fluid ions and Boltzmann electrons, is to avoid getting lost in the purely algebraic details of more complicated plasma and wave models, and to stress that already at this level the Maxon-Viecelli results are of limited use.

A perusal of the graphs in the literature shows that most of them are drawn in such a way that one is dealing with inward propagation, as advocated here, but almost none specify this, and it is very difficult to assess this from the choices of the stretching in $\xi$ and $\tau$.

Unfortunately, only about half of the papers quoted here and in our earlier discussion ${ }^{12}$ respect the limit on the amplitudes $\left(n_{1}\right.$ or $\varphi_{1} \simeq 0.1$, whether it be in the initial value chosen for substitution in (33) to start the numerical solution or at the point where the graphs stop.

More importantly, as explained above, the limit quoted by Maxon and Viecelli on the minimum admissible $r$ is intimately connected to the adopted amplitudes. The latter, however, are intrinsically given by a general feature of the reductive perturbation method. In the light of this, it is extremely disappointing to see that none of the papers investigated even remotely respects the minimum limit on $r$, and consequently, also on $|\tau|$. The sad conclusion is that all the papers that we have surveyed breach the Maxon-Viecelli limits in one or two ways, including even the original papers themselves.

From the exposition given here and from the many examples in the literature, it is clear that reductive perturbation theory can be adapted to the investigation of many other nonplanar waves, describable by extensions of the Burgers, ${ }^{43}$ nonlinear Schrödinger ${ }^{44}$ Kadomtsev-Petviashvili, ${ }^{45}$ ZakharovKuznetsov $^{46}$ equations and many more. It would be unwieldy to discuss all these in any reasonable detail, which would lead to an overlong and unmanageable reference list. Moreover, the plasma composition can also be extended in many directions and still be handled in the framework discussed here.

Another aspect to be discussed is how the graphs illustrating the different papers have been generated. As in the previous roundup, ${ }^{12}$ where it was an overwhelming majority, all papers now reviewed ${ }^{18,27-42}$ bar one ${ }^{26}$ state that there is no exact solution to (34), and therefore, the one-soliton solution (33) (or a suitable variation thereof) has been used as an acceptable initial profile at large $|\tau|$ (where supposedly $\left.\nu \varphi_{1} / 2 \tau \rightarrow 0\right)$ and then advanced towards smaller $|\tau|$, towards the axis or centre of symmetry.

There are serious problems with this approach. The initial choices of what is called large $|\tau|$ are all $|\tau| \leq 30$, chosen below or at the limit of a range where (34) is not valid, given the approximations made to derive it. More fundamental is that at $|\tau| \leq 30$ one starts from the plane mode given in (33), 
which is then advanced under (34) deeper into the forbidden domain, so that any result, even if numerically correct, totally lacks credibility.

There are a couple of papers in the literature giving exact solutions of the nonplanar $\mathrm{KdV}$ equation, ${ }^{26,47,48}$ but these solutions are obtained through a series of transformations, are not at all what one could recognize as a solitary wave of fixed form or of fixed speed, and are very difficult to plot in a simple way in the original variables used in (34). In addition, it is claimed ${ }^{47}$ that even at very large $|\tau|$ these exact solutions differ from the traditional sech-squared soliton, seemingly implying that this is not a good choice to start the numerical evaluations with.

The problem with the exact solutions is in a way reminiscent of what happens with the standard $\mathrm{KdV}$ equation. If one considers the KdV equation as a given mathematical entity, one can try to extract all possible information as to invariants, soliton solutions, interaction properties, and so on. However, if one approaches the KdV equation from a physical point of view, it is derived in plasma physics through the reductive perturbation method, which truncates the intrinsically fully nonlinear basic equations to a certain order. It seems then logical, but is often not implemented, that the solutions of the KdV equation for a particular model can only be valid from a physics point of view if they obey the underlying restrictions. Clearly, the mathematical discussion of the KdV equation and its properties does not care about such restrictions, but the physicists must heed them.

The paper by Ghosh et al. ${ }^{26}$ is an illustration of the previous remarks: the exact solution (38) of their extended KdV equation (12) is based on the Hirota transform. ${ }^{48}$ However, as the plot in their Fig. 6 indicates, the amplitudes are far too large and the normalized time goes from -10 to +10 , right through zero without any infinity there, although it is well known that any solution of the extended $\mathrm{KdV}$ equation becomes infinite when $\tau \rightarrow 0$ (in our notation, $\eta$ in theirs).

\section{CONCLUSIONS}

In recent years, we have observed the appearance of numerous papers on weakly nonlinear nonplanar solitons that appear to have a cavalier attitude towards the limitations imposed by the underlying assumptions of the formalism used. Hence, we have presented a thorough analysis of the basis and the implications of the stretching and related aspects of the technique pioneered by Maxon and Viecelli ${ }^{5,6}$ in their development and interpretation of an extended Korteweg-de Vries (eKdV) equation:

(1) We have shown unequivocally that the stretching used by Maxon and Viecelli for nonplanar structures ${ }^{5,6,13,14}$ implies severe restrictions on the spatial domain where the resulting eKdV equation can produce acceptable results, even though the stretching looks deceptively simple, copying essentially the standard plane-wave stretching by replacing a cartesian by a radial coordinate. The most stringent restriction is the one which is not obeyed at all in the literature, as far as the applications are concerned: one has to keep a minimum distance away from the axis or centre of symmetry for cylindrical or spherical modes, respectively. At closer distances, the eKdV equation is not valid.

(2) This minimum distance is intrinsically tied to the weakly nonlinear amplitudes acceptable under the reductive perturbation analysis used to derive the eKdV equation. This is not new, as already noted by Maxon and Viecelli themselves, ${ }^{6,13,14}$ although universally ignored.

(3) Going through the algebra one arrives at an eKdV equation, extending the well-known $\mathrm{KdV}$ equation with a term which is linear in $\varphi_{1}$ and inversely proportional to the slow time $\tau$. The proper derivation leads to a term in $\varphi_{1} / r$, indicating the restriction in $r$. This is then transferred to a limitation on $\tau$ by combining the elements of the stretching, a procedure which obscures the spatial origin of the restriction.

(4) The eKdV equation (34) has no soliton solution in the accepted sense, and no direct exact solution. Instead, there are procedures in the literature which reduce the $\mathrm{eKdV}$ equation to a standard $\mathrm{KdV}$ equation through a series of coordinate transformations. ${ }^{47,48}$ Transforming back to the original variables $\xi$ and $\tau$ yields a mathematically correct solution, if the eKdV equation were given. Unfortunately, however, it does not satisfy the restrictions placed on the validity of the eKdV equation as derived in a given context, and hence is physically not acceptable.

(5) Therefore, almost all papers use for the visualisation of the soliton profiles, the usual sech-squared one-soliton solution as an initial profile, at a supposedly large distance away from the axis or centre of geometry, where the structures are assumed quasi-planar. For more complicated plasma compositions and nonlinear evolution equations the procedure is analogous, mutatis mutandis. However, since the original starting distance is unfortunately already at or below the minimum forced by the restrictions imposed by the analytical derivation of (34), the resulting graphs make no sense: the evolution of the initial sech-squared soliton is governed by an equation which is not valid in the domain covered by the figures.

(6) The upshot of all this is that we do not really know how nonplanar modes would look like and evolve when propagating towards the singularity at $\tau=0$ or $r=0$, except that the amplitude would blow up. At the minimum distances imposed by the modified reductive perturbation analysis the structures look very much quasi-planar, and thus, the new physically correct information of this part of the literature is meagre. We thus repeat one of our earlier conclusions ${ }^{12}$ that this is a problem which would be well served by a serious numerical simulation, not starting from the restricted $\mathrm{eKdV}$ equation but directly from the basic equations, without approximations.

\section{ACKNOWLEDGMENTS}

The authors are grateful to an anonymous referee for some helpful suggestions. M.A.H. thanks the National Research Foundation of South Africa for partial support under Grant No. 68911. Any opinions, findings and conclusions expressed herein are those of the authors, and the NRF accepts no liability whatsoever in this regard. 
${ }^{1}$ D. J. Korteweg and G. de Vries, Philos. Mag. 39, 422 (1895).

${ }^{2}$ H. Washimi and T. Taniuti, Phys. Rev. Lett. 17, 996 (1966).

${ }^{3}$ P. G. Drazin and R. S. Johnson, Solitons: An Introduction (Cambridge University Press, Cambridge, UK, 1989).

${ }^{4}$ R. C. Davidson, Methods in Nonlinear Plasma Theory (Academic Press, New York, 1972).

${ }^{5}$ S. Maxon and J. Viecelli, Phys. Rev. Lett. 32, 4 (1974).

${ }^{6}$ S. Maxon and J. Viecelli, Phys. Fluids 17, 1614 (1974).

${ }^{7}$ N. Hershkowitz and T. Romesser, Phys. Rev. Lett. 32, 581 (1974)

${ }^{8}$ Y. Nakamura, M. Ooyama, and T. Ogino, Phys. Rev. Lett. 45, 1565 (1980).

${ }^{9}$ I. Tsukabayashi, Y. Nakamura, and T. Ogino, Phys. Lett. A 81, 507 (1981).

${ }^{10}$ Y. Nakamura and T. Ogino, Plasma Phys. 24, 1295 (1982).

${ }^{11}$ Y. Nakamura, IEEE Trans. Plasma Sci. 10, 180 (1982).

${ }^{12}$ F. Verheest and M. A. Hellberg, Phys. Plasmas 21, 022307 (2014).

${ }^{13}$ S. Maxon, Phys. Fluids 19, 266 (1976).

${ }^{14}$ S. Maxon, Rocky Mt. J. Math. 8, 269 (1978).

${ }^{15}$ F. F. Chen, Introduction to Plasma Physics and Controlled Fusion, Vol. 1: Plasma Physics, 2nd ed. (Plenum Press, New York, 1984).

${ }^{16}$ B. Leroy, Eur. J. Phys. 10, 82 (1989).

${ }^{17}$ W. R. Lepage, Complex Variables and the Laplace Transform for Engineers (McGraw-Hill, New York, 1961), pp. 336-338.

${ }^{18}$ S. A. Shan and A. Rehman, Astrophys. Space Sci. 352, 593 (2014).

${ }^{19}$ S. A. Shan and A. Rehman, Astrophys. Space Sci. 360, 70 (2015).

${ }^{20}$ J. D. Jackson, Classical Electrodynamics, 3rd ed. (Wiley, New York, 1998), pp. 425-429.

${ }^{21}$ R. Courant, in Modern Mathematics for the Engineer, edited by E. F. Beckenbach (McGraw-Hill, New York, 1956), p. 92.

${ }^{22}$ A. Sommerfeld, Optics (Academic Press, New York, 1964).

${ }^{23}$ P. M. Morse and H. Feshbach, Methods of Theoretical Physics (McGraw Hill, New York, 1953).

${ }^{24}$ E. Infeld and G. Rowlands, Nonlinear Waves, Solitons and Chaos (Cambridge University Press, Cambridge, UK, 2000).

${ }^{25} \mathrm{We}$ thank an anonymous referee for drawing this argument to our attention.
${ }^{26}$ S. K. Ghosh, S. K. Gupta, and P. Chatterjee, Phys. Scr. 90, 125601 (2015).

${ }^{27}$ J.-N. Han, J.-H. Luo, and J.-X. Li, Astrophys. Space Sci. 349, 305 (2014).

${ }^{28}$ J.-N. Han, W.-S. Duan, J.-X. Li, Y.-L. He, J.-H. Luo, Y.-G. Nan, Z.-H. Han, and G.-X. Dong, Phys. Plasmas 21, 012102 (2014).

${ }^{29}$ J.-N. Han, J.-X. Li, J.-H. Luo, G.-H. Sun, Z.-L. Liu, S.-H. Ge, and X.-X. Wang, Phys. Scr. 89, 025603 (2014).

${ }^{30}$ I. Tasnim, M. M. Masud, and A. A. Mamun, J. Korean Phys. Soc. 64, 987 (2014).

${ }^{31}$ H. R. Pakzad, K. Javidan, and M. Tribeche, Astrophys. Space Sci. 352, 185 (2014).

${ }^{32}$ M. M. Rahman, M. S. Alam, and A. A. Mamun, Astrophys. Space Sci. 352, 193 (2014).

${ }^{33}$ M. R. Hossen, L. Nahar, S. Sultana, and A. A. Mamun, Astrophys. Space Sci. 353, 123 (2014).

${ }^{34}$ S. A. Shan, S. Ali, and Aman-ur-Rehman, Astrophys. Space Sci. 353, 151 (2014).

${ }^{35}$ M. R. Hossen and A. A. Mamun, Braz. J. Phys. 44, 673 (2014).

${ }^{36}$ M. R. Hossen, L. Nahar, and A. A. Mamun, J. Korean Phys. Soc. 65, 1863 (2014).

${ }^{37}$ M. R. Hossen and A. A. Mamun, J. Korean Phys. Soc. 65, 2045 (2014).

${ }^{38}$ P. K. Mandal, M. K. Ghorui, A. Saha, and P. Chatterjee, Astrophys. Space Sci. 355, 89 (2015).

${ }^{39}$ M. J. Uddin, M. S. Alam, and A. A. Mamun, Phys. Plasmas 22, 022111 (2015).

${ }^{40}$ M. G. Shah, A. A. Mamun, and M. R. Hossen, J. Korean Phys. Soc. 66, 1239 (2015).

${ }^{41}$ A. A. Mamun and F. Deeba, Plasma Phys. Rep. 41, 667 (2015).

${ }^{42}$ S. A. Ema, M. R. Hossen, and A. A. Mamun, Contrib. Plasma Phys. 55, 596 (2015)

${ }^{43}$ J. M. Burgers, Adv. Appl. Mech. 1, 171 (1948).

${ }^{44}$ T. Kakutani, H. Ono, T. Taniuti, and C.-C. Wei, J. Phys. Soc. Jpn. 24, 1159 (1968).

${ }^{45}$ B. B. Kadomtsev and V. I. Petviashvili, Sov. Phys. JETP 16, 1578 (1963).

${ }^{46}$ V. E. Zakharov and E. A. Kuznetsov, Sov. Phys. JETP 39, 285 (1974).

${ }^{47}$ F. Calogero and A. Degasperis, Lett. Nuovo Cimento 23, 150 (1978).

${ }^{48}$ R. Hirota, Phys. Lett. A 71, 393 (1979). 